\title{
Fundamentals of multidimensional multirate digital signal processing
}

\author{
P P VAIDYANATHAN \\ Department of Electrical Engineering, California Institute of Technology, \\ Pasadena, CA 91125, USA
}

\begin{abstract}
This paper reviews the fundamentals of multidimensional multirate signal processing. Central to these discussions is the idea of generalized sampling-lattice. Topics discussed include nonrectangular decimators, interpolators, generalized DFT, and filter banks. The multidimensional polyphase decomposition is developed, with applications in decimation filtering and perfect reconstruction filter banks.
\end{abstract}

Keywords. Multidimensional filter-banks; lattice decimation; polyphase structures; multidimensional sampling; multirate systems.

\section{Introduction}

In multirate digital signal processing, the sampling rate is allowed to vary from point to point, by use of building blocks called decimators and interpolators (Crochiere \& Rabiner 1981, 1983; Vaidyanathan 1990). Typical applications in one-dimensional (1-D) signal processing include digital audio systems, subband coding of speech (Crochiere 1975) and music (Fettweis et al 1990), and voice privacy systems. We refer the reader to Vaidyanathan (1990) and references therein for details on 1-D multirate systems. Multirate ideas have recently been extended to two and higher dimensional systems by a number of authors (Vetterli 1984; Wackersreuther 1986; Ansari \& Lau 1987; Vaidyanathan 1987; Viscito \& Allebach 1988; Ansari \& Guillemot 1990; Bamberger \& Smith 1990; Karlsson \& Vetterli 1990), with typical applications in image compression and coding. Thus multidimensional (MD) versions of decimation and interpolation filters, maximally decimated filter banks, polyphase decompositions and perfect reconstruction systems have already been proposed. The basic building blocks here are decimators, interpolators, and digital filters.

In this paper we provide a fairly thorough treatment of fundamentals of multidimensional multirate systems. Unless mentioned otherwise, our discussions here hold for general $D$-dimensional systems. We shall deal with MD sequences such as $x(n)$ where $\boldsymbol{n}$ is a $D$-dimensional integer vector (called the 'time-index' for convenience). For such systems, decimators and interpolators are defined in terms of integer matrices. For example an $\mathbf{M}$-fold decimator is defined by the input-output relation $y(\mathbf{n})=x(\mathbf{M n})$, where $\mathbf{M}$ is a $D \times D$ nonsingular integer matrix. If $\mathbf{M}$ happens to be diagonal we say

A list of special notation used is given at the end of the paper. 
that the decimator is rectangular. (Similar jargon applies to interpolators; see below.) In general, when decimators and interpolators are rectangular, the filters associated with them are separable filters [i.e., the transfer functions are products of 1-D filters in separate dimensions, as in $\left.H_{2}\left(z_{0}, z_{1}\right)=H_{0}\left(z_{0}\right) H_{1}\left(z_{1}\right)\right]$. These can then be designed easily by use of $1-D$ methods. Similarly, in a multidimensional maximally decimated filter bank $(\$ 7)$ if the decimators and interpolators are rectangular, then 1-D filters can be used to generate the MD separable filters. In this way, one-dimensional aliasfree systems and perfect reconstruction systems can be translated (Vaidyanathan 1987). In practice, however, there is greater interest in nonrectangular decimators and interpolators (Ansari \& Lau 1987; Viscito \& Allebach 1988; Ansari \& Guillemot 1990; Bamberger \& Smith 1990; Karlsson \& Vetterli 1990), which is what we shall consider.

Paper outline: After a brief review of multidimensional Fourier transform notation in $\S 2$, we introduce lattices in $\S 3$. These find application in multidimensional decimation and interpolation which are discussed in $\$ 4$. Section 5 introduces the polyphase decomposition. In $\$ 6$ we discuss the generalized orthogonal exponentials and a generalized form of the DFT matrix, which is more suitable for multirate work. Maximally decimated filter banks are discussed in $\$ 7$.

Notations: Boldface letters denote matrices and vectors. $\mathbf{A}^{\boldsymbol{T}}$ and $\mathbf{A}^{\dagger}$ denote the transpose and transposed-conjugate of $\mathbf{A}$, respectively. The row and column indices typically begin from zero. The notation $[a, b)^{D}$ for real $a, b$ stands for the set of all $D \times 1$ real vectors with components $x_{i}$ in the range $a \leqslant x_{i}<b$. [The set $(a, b)^{D}$ is similarly defined, but now $a<x_{i}<b$.] The notation $\mathscr{N}$ stands for the set of all $D \times 1$ integers. The determinant of a square matrix is abbreviated as det $M$. In $\S 8$ all new notations will be summarized.

\section{Multidimensional Fourier transform}

\subsection{Continuous-time signals}

A $D$-dimensional signal $x_{a}\left(t_{0}, t_{1}, \ldots t_{D-1}\right)$ is a function of $D$ real variables $t_{0}, t_{1}, \ldots t_{D-1}$. We shall define the column vector

$$
t=\left[t_{0} t_{1} \ldots t_{D-1}\right]^{T}
$$

and abbreviate the signal as $x_{a}(t)$. The subscript $a$ indicates 'analog' which actually means that $t_{i}$ are continuous variables. Even though these variables do not (necessarily) represent time, it is customary to call them so, and refer to $x_{a}(t)$ as a continuous 'time' signal. Its Fourier transform (if it exists) is denoted as $X_{a}(j \Omega)$. The relation between $x_{a}(t)$ and $X_{a}(j \Omega)$ is

where

$$
\begin{aligned}
X_{a}(j \Omega) & =\int_{-\infty}^{\infty} x_{a}(t) \exp \left[-j \Omega^{T} \mathrm{t}\right] \mathrm{dt}, \\
x_{a}(t) & =\left[1 /(2 \pi)^{D}\right] \int_{-\infty}^{\infty} X_{a}(j \Omega) \exp \left[j \mathbf{\Omega}^{T} t\right] \mathrm{d} \Omega,
\end{aligned}
$$

$$
\Omega=\left[\Omega_{0} \Omega_{1} \ldots \Omega_{D-1}\right]^{T} \text {. }
$$



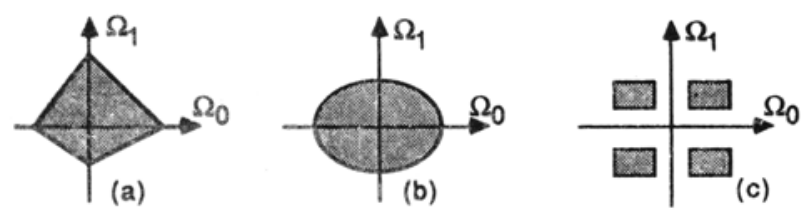

Figure 1. Examples of frequency domain supports for bandlimited signals. (a) and (b) are lowpass signals; (c) is a bandpass filter.

The notation $\int_{a}^{b}$ indicates that the integration is with respect to all variables, with each variable ranging from $a$ to $b$. With $D=1$ the above relations reduce to familiar 1-D relations.

Bandlimited signals: $x_{a}(t)$ is said to be bandlimited if $X_{a}(j \Omega)$ is identically zero everywhere except in a designated finite region. The region where $X_{a}(j \Omega)$ is allowed to be nonzero is said to be the support of $X_{a}(j \Omega)$. Figure 1 shows some examples, with gray areas indicating the support. The examples in parts (a) and (b) are lowpass signals; if $\left|X_{a}(j \Omega)\right|$ is a nonzero constant in the gray areas, it can be considered to be a lowpass fiiter [i.e., $x_{a}(t)$ is the impulse response of a 2-D lowpass filter]. Part (c), on the other hand, would represent a bandpass filter.

\subsection{Discrete-time signals}

$D$-dimensional sequences are denoted as $x(\mathbf{n}), y(\mathbf{n})$, and so on, where $\mathbf{n}$ is the $D \times 1$ vector of integers $\mathbf{n}=\left[n_{0} n_{1} \ldots n_{D-1}\right]^{T}$. Denoting the Fourier transform of $x(\mathbf{n})$ by $X(\omega)$, we have

$$
X(\boldsymbol{\omega})=\sum_{\mathbf{n} \in 1^{1}} x(\mathbf{n}) \exp \left[-j \boldsymbol{\omega}^{T} \mathbf{n}\right], \quad x(\mathbf{n})=\left[1 /(2 \pi)^{D}\right] \int_{-\pi}^{\pi} X(\boldsymbol{\omega}) \exp \left(j \omega^{T} \mathbf{n}\right) \mathrm{d} \boldsymbol{\omega}
$$

The frequency vector $\omega$ is $D$-dimensional, i.e., $\omega=\left[\omega_{0} \omega_{1} \ldots \omega_{D-1}\right]^{T}$. The integral and summation above are $D$-dimensional. The function $X(\omega)$ is periodic in $\omega$ with periodicity matrix $2 \pi \mathbf{I}$. [A multidimensional function $f(\omega)$ is said to be periodic (Dudgeon \& Mersereau 1984) with periodicity matrix $\mathbf{P}$ if $f(\boldsymbol{\omega}+\mathbf{P k})=f(\boldsymbol{\omega})$ for all $k \in \mathscr{N}$.]

The $\mathscr{Z}$-transform of $x(\mathbf{n})$, where it converges, is given by

where

$$
X_{z}(\mathbf{z})=\sum_{\mathbf{n} \in \mathbf{4}}^{1} x(\mathbf{n}) \mathscr{Z}(-\mathbf{n})
$$

and

$$
\mathbf{z}=\left[z_{0} z_{1} \ldots z_{D-1}\right]^{T}
$$

$$
\not \mathbf{Z}(\mathbf{n}) \triangleq z_{0}^{n_{0}} z_{1}^{n_{1}} \ldots z_{D-1}^{n_{D-1}}
$$

Note the subscript $z$ on the $X$ which is used in (5), but not in (4). The quantity $\mathscr{2}(-\mathbf{k})$ represents a delay operator. More specifically, the $\mathscr{Z}$-transform of $x(\mathbf{n}-\mathbf{k})$ is $\mathscr{Z}(-\mathbf{k}) X_{z}(\mathbf{z})$. Also note the properties $\mathscr{Z}(\mathbf{k})=1 / \mathscr{Z}(-\mathbf{k})$, and $\mathscr{Z}(\mathbf{k}+\mathbf{m})=\mathscr{Z}(\mathbf{k}) \mathscr{Z}(\mathbf{m})$. For steady state frequencies, $z(-\mathbf{k})=\exp \left[-j \omega^{T} \mathbf{k}\right]$.

\section{Lattices}

Let $\mathbf{M}$ be any $D \times D$ real nonsingular matrix. The lattice $L A T(\mathbf{M})$ generated by $\mathbf{M}$ is the sei of all $D \times 1$ vectors of the form $\mathbf{M n}$ where $\mathbf{n}$ varies over the set $\mathscr{N}$ of all 


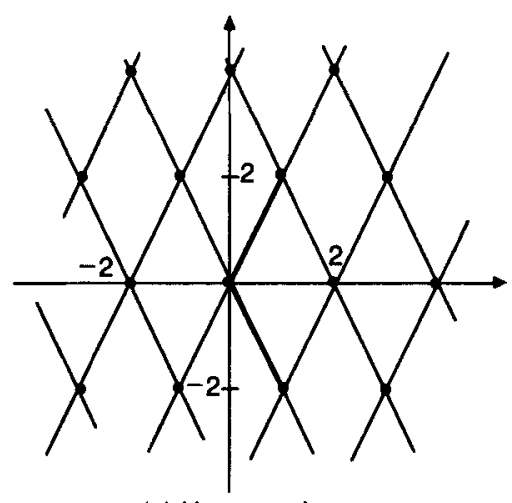

(a) Hexagonal

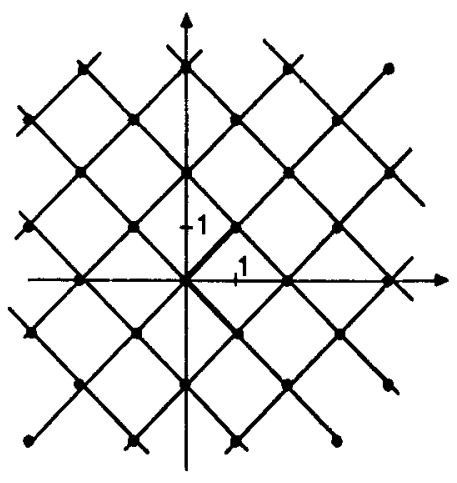

(b) Quincunx

Figure 2. Examples of lattices. (a) Hexagonal lattice, and (b) quincunx lattice.

integers. Thus the lattice is the set of all integer linear combinations of columns of M. Figure 2 demonstrates the lattices for

$$
\mathbf{M}=\left[\begin{array}{rr}
1 & 1 \\
2 & -2
\end{array}\right] \text { (hexagonal), and } \mathbf{M}=\left[\begin{array}{rr}
1 & 1 \\
-1 & 1
\end{array}\right] \text { (quincunx), }
$$

where the lattice points are indicated by black dots. These are two commonly used examples of decimators in two-dimensional systems (Ansari \& Lau 1987; Ansari \& Guillemot 1990; Bamberger \& Smith 1990; Karlsson \& Vetterli 1990) and are respectively called the hexagonal and quincunx matrices. From figure 2 we see that the points on the lattice are at the intersections of two sets of parallel, equispaced, lines. The orientations of these lines coincide with the orientations of the vectors (heavy lines in the figure) which form the colums of $\mathbf{M}$.

Invariance under unimodular change of basis: It turns out (Cassels 1959; Dubois 1985) that the lattice is unchanged if the generator $\mathbf{M}$ is replaced with $M U$ where $U$ is a unimodular integer matrix, i.e., integer matrix with det $\mathrm{U}= \pm 1$. So $L A T(\mathbf{M})=$ $L A T(\mathbf{M U})$. For example, the matrix

$$
\underbrace{\left[\begin{array}{rr}
1 & 1 \\
-1 & 1
\end{array}\right]}_{\text {quincunx } \mathbf{M}} \underbrace{\left[\begin{array}{ll}
1 & 0 \\
1 & 1
\end{array}\right]}_{\mathbf{U}}=\left[\begin{array}{ll}
2 & 1 \\
0 & 1
\end{array}\right],
$$

generates the same lattice as does the quincunx matrix.

Fundamental and symmetric parallelopipeds: Given the matrix $\mathbf{M}$, we associate with it a set of real vectors called the fundamental parallelopiped ((FPD(M)) defined as

$$
\operatorname{FPD}(\mathbf{M})=\text { set of all vectors } \mathbf{M x} \text {, with } \mathbf{x} \in[0,1)^{D} \text {. }
$$

We shall also define a set called the symmetric parallelopiped $(\operatorname{SPD}(\mathbf{M}))$ as

$$
\operatorname{SPD}(\mathbf{M})=\text { set of all vectors } \mathbf{M x} \text {, with } \mathbf{x} \in[-1,1)^{D} \text {. }
$$

The region FPD(M) has hypervolume (area in $2-\mathrm{D}$ case) equal to $|\operatorname{det} \mathbf{M}|$. Figure 3 


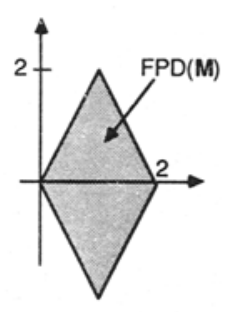

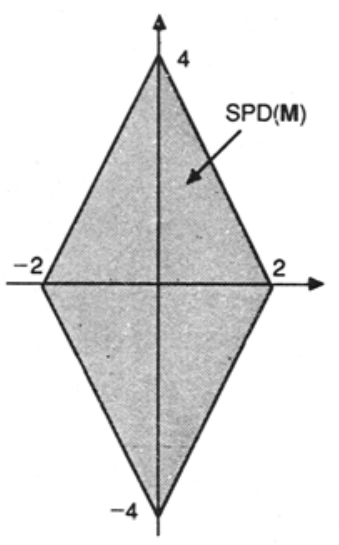

Figure 3. Fundamental and symmetric parallelopipeds for hexagonal $\mathbf{M}$.

demonstrates $\mathrm{FPD}(\mathbf{M})$ and $\operatorname{SPD}(\mathbf{M})$ for the hexagonal matrix defined in (7). It is easily verified that $\operatorname{SPD}(\mathbf{M})$ and $\operatorname{FPD}(\mathbf{M})$ are related as follows:

$$
\operatorname{SPD}(\mathbf{M})=\operatorname{FPD}(2 \mathbf{M})-\mathbf{M}\left[\begin{array}{c}
1 \\
\vdots \\
1
\end{array}\right]
$$

Thus $\operatorname{SPD}(\mathbf{M})$ is a shifted version of a scaled version of $\operatorname{FPD}(\mathbf{M})$. This is clearly seen in figure 3.

\subsection{Integer lattices}

In multirate work, the matrix $\mathbf{M}$ is further restricted to have integer entries. In all discussions to follow, $\mathbf{M}$ denotes a $D \times D$ nonsingular integer matrix unless mentioned otherwise. The elements of $L A T(\mathbf{M})$ are themselves integers. The integer $|\operatorname{det} \mathbf{M}|$, which will play a crucial role in our discussion, is given the notation

$$
J(\mathbf{M}) \triangleq|\operatorname{det} \mathbf{M}| \text {. }
$$

Since this is the hypervolume of $\operatorname{FPD}(\mathbf{M})$, there are precisely $J(\mathbf{M})$ integer vectors in FPD $(\mathbf{M})$. This set of $J(\mathbf{M})$ integers is denoted as $\mathscr{N}(\mathbf{M})$, i.e.,

$$
\mathcal{N}(\mathbf{M})=\text { set of all integers } \mathbf{M x} \text {, with } \mathbf{x} \in[0,1)^{D} \text {. }
$$

This is demonstrated in figure 4 where $\operatorname{FPD}(\mathbf{M})$ is shown for hexagonal $\mathbf{M}$. Since $J(M)=4$, we have four integers in $\mathrm{FPD}(M)$ (indicated by black dots). Thus

$$
\text { set } \mathscr{N}(\mathbf{M}): \mathbf{k}_{0}=\left[\begin{array}{l}
0 \\
0
\end{array}\right], \mathbf{k}_{1}=\left[\begin{array}{l}
1 \\
0
\end{array}\right], \mathbf{k}_{2}=\left[\begin{array}{l}
1 \\
1
\end{array}\right], \mathbf{k}_{3}=\left[\begin{array}{r}
1 \\
-1
\end{array}\right](\text { hexagonal } \mathbf{M}) \text {. }
$$

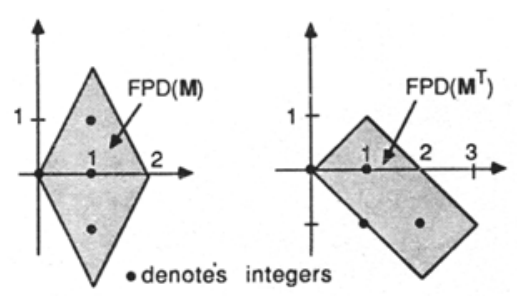

Figure 4. Integers falling inside fundamental parallelopipeds. $M$ is hexagonal. 
The figure also shows $\operatorname{FD}\left(\mathbf{M}^{T}\right)$ and the set $\mathcal{N}\left(\mathbf{M}^{T}\right)$ of integers inside it, for future use. Thus

$$
\text { set } \begin{aligned}
\mathscr{N}\left(\mathbf{M}^{T}\right): \mathbf{m}_{0} & =\left[\begin{array}{l}
0 \\
0
\end{array}\right], \mathbf{m}_{1}=\left[\begin{array}{l}
1 \\
0
\end{array}\right], \mathbf{m}_{2}=\left[\begin{array}{r}
1 \\
-1
\end{array}\right], \\
\mathbf{m}_{3} & \left.=\left[\begin{array}{r}
2 \\
-1
\end{array}\right] \text { (hexagonal } \mathbf{M}\right) .
\end{aligned}
$$

Division theorem for integer vectors: Given any integer vector $\mathbf{n}$ we can always write

$$
\mathbf{n}=\mathbf{k}+\mathbf{M} \mathbf{n}_{0}, \quad \mathbf{k}, \mathbf{n}_{0} \text { unique integers, with } \mathbf{k} \in \mathcal{N}(\mathbf{M}) .
$$

This is analogous to the division theorem for integers. [Sketch of proof. Define $\mathbf{y}=\mathbf{M}^{-1} \mathbf{n}$. Each component of this vector can be written as a unique integer plus nonnegative fraction in the range $[0,1)$. Thus $y=x+n_{0}$ where $x \in[0,1)^{D}$ and $n_{0} \in \mathscr{N}$. But $\mathbf{n}=$ My so that (15a) follows.]

We say that $\mathbf{k}$ is the remainder of $\mathbf{n}$ modulo $\mathbf{M}$. And $\mathbf{n}_{\mathbf{0}}$ is called the quotient. Other notations to indicate this quantity $\mathbf{k}$ are:

$$
\mathbf{k}=\mathbf{n} \bmod \mathbf{M} \text {, and } \mathbf{k}=((\mathbf{n}))_{M} .
$$

Note that the set $\mathcal{N}$ of all integer vectors can be partitioned into $J(\mathbf{M})$ sets [called cosets of the lattice $L A T(\mathbf{M})$, (Cassels 1959; Dubois 1985)], each set being characterized by one distinct value of $\mathbf{k} \in \mathscr{N}(\mathbf{M})$.

\subsection{Lattice-sampling of a continuous time signal}

Let $x_{a}(t)$ be a $D$-dimensional signal with Fourier transform $X_{a}(j \Omega)$. Define the sequence $x(\mathbf{n})=x_{a}(\mathbf{V n})$, where $\mathrm{V}$ is $D \times D$ real nonsingular. $x(\mathbf{n})$ is called the sampled version of $x_{a}(t)$, and $V$ is called the sampling matrix. Denote the Fourier transforms of $x(n)$ and $x_{a}(t)$ by $X(\omega)$ and $X_{a}(j \Omega)$ respectively. It is well-known (Dudgeon \& Mersereau 1984) that these are related as

$$
\begin{aligned}
X(\omega) & =\frac{1}{|\operatorname{det} \mathbf{V}|} \sum_{\mathbf{k} \in 1^{\circ}} X_{\mathbf{a}}\left(j \mathbf{V}^{-T}(\omega-2 \pi \mathbf{k})\right) \\
& =\frac{1}{|\operatorname{det} \mathbf{V}|} \sum_{\mathbf{k} \in 1^{\circ}} X_{a}\left(j\left(\Omega-2 \pi \mathbf{V}^{-T} \mathbf{k}\right)\right), \quad \Omega=\mathbf{V}^{-T} \omega .
\end{aligned}
$$

With $\mathbf{V}=T(1-\mathrm{D}$ case), the RHS reduces to the familiar 1-D result (Oppenheim \& Schafer 1975). The lattice $L A T\left(V^{-T}\right)$ is said to be the reciprocal lattice of $L A T(V)$. And $L A T\left(2 \pi \mathbf{V}^{-T}\right)$ is the scaled reciprocal lattice.

The shifted versions of $X_{a}(j \Omega)$ (i.e., terms in (16) with $\mathbf{k} \neq 0$ ) are called alias components. If any of these has overlap with the unshifted version, we cannot recover $X_{a}(j \Omega)$ from $X(\omega)$. This is similar to the aliasing effect in the 1-D case. If the signal $X_{a}(j \Omega)$ is 'appropriately bandlimited', and if the sampling matrix $\mathbf{V}$ is appropriately chosen, this overlap can be avoided. 


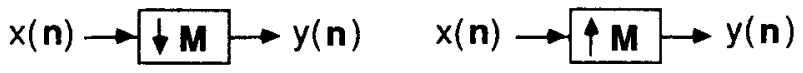

(a) Decimator

(b) Interpolator

Figure 5. Multidimensional multirate building blocks.

\section{Decimators and interpolators}

Figure 5 shows block diagrams for M-fold decimators and interpolators. The decimator has the input-output relation

$$
y(\mathbf{n})=x(\mathbf{M n}),
$$

so that only the sample points which lie on the lattice $L A T(M)$ are retained. The interpolator has the input-output relation

$$
y(\mathbf{n})= \begin{cases}x\left(\mathbf{M}^{-1} \mathbf{n}\right), & \mathbf{n} \in \operatorname{LAT}(\mathbf{M}) \\ 0 & \text { otherwise }\end{cases}
$$

In other words, for every integer $\mathbf{n}$, we have $y(\mathbf{M n})=x(\mathbf{n})$. Those samples of $y(\mathbf{n})$ which do not fall at the points of $\operatorname{LAT}(\mathbf{M})$ are set to zero.

Decimation ratio: We know there are precisely $|\operatorname{det} \mathbf{M}|$ integers in $\operatorname{FPD}(\mathbf{M})$. This means that, on the average one out of $|\operatorname{det} \mathbf{M}|$ sample points are retained by the decimator so that the decimation ratio is $|\operatorname{det} \mathbf{M}|$.

\subsection{Transform domain expressions}

The effect of decimator in the transform domain is expressed by the relation

$$
Y(\omega)=[1 / J(\mathbf{M})] \sum_{\mathbf{k} \in t^{\prime}\left(\mathbf{M}^{\tau}\right)} X\left(\mathbf{M}^{-M}(\omega-2 \pi \mathbf{k})\right) \quad(\mathbf{M} \text {-fold decimator })
$$

Proof. We shall derive this by assuming that $x(\mathbf{n})$ has been obtained by sampling an analog signal $x_{a}(t)$ with some sampling matrix $V$, i.e., $x(\mathbf{n})=x_{a}(\mathbf{V n})$. (Proofs which do not make this assumption exist.) We know that $X(\omega)$ and $X_{a}(j \Omega)$ are related as in (16). Evidently $y(\mathbf{n})$ is obtainable directly from $x_{a}(\mathbf{t})$ as $y(\mathbf{n})=x_{a}(\mathbf{V M n})$. So

$$
Y(\boldsymbol{\omega})=(1 /|\operatorname{det} \mathbf{V M}|) \sum_{\mathbf{n} \in I^{-}} X_{a}\left(j \mathbf{V}^{-T} \mathbf{M}^{-T}(\boldsymbol{\omega}-2 \pi \mathbf{n})\right) .
$$

We now use the division theorem to write the summation index $n$ as $n=M^{T} n_{0}+k$, with $k \in \mathscr{N}\left(\mathbf{M}^{T}\right)$ and $\mathbf{n}_{0} \in \mathscr{N}$. Thus $Y(\omega)$ can be rearranged as

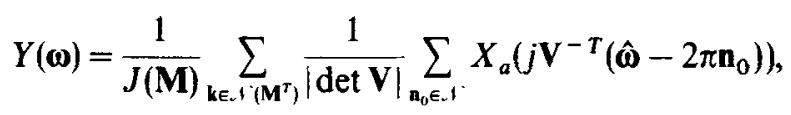

where $\hat{\boldsymbol{\omega}}=\mathbf{M}^{-T}(\boldsymbol{\omega}-2 \pi \mathbf{k})$. The inner summation can be written in terms of $X(\boldsymbol{\omega})$ so that $Y(\omega)$ reduces to $(19)$ indeed.

Aliasing due to decimation: The term in (19) with $\mathbf{k}=\mathbf{0}$ represents the 'stretched 


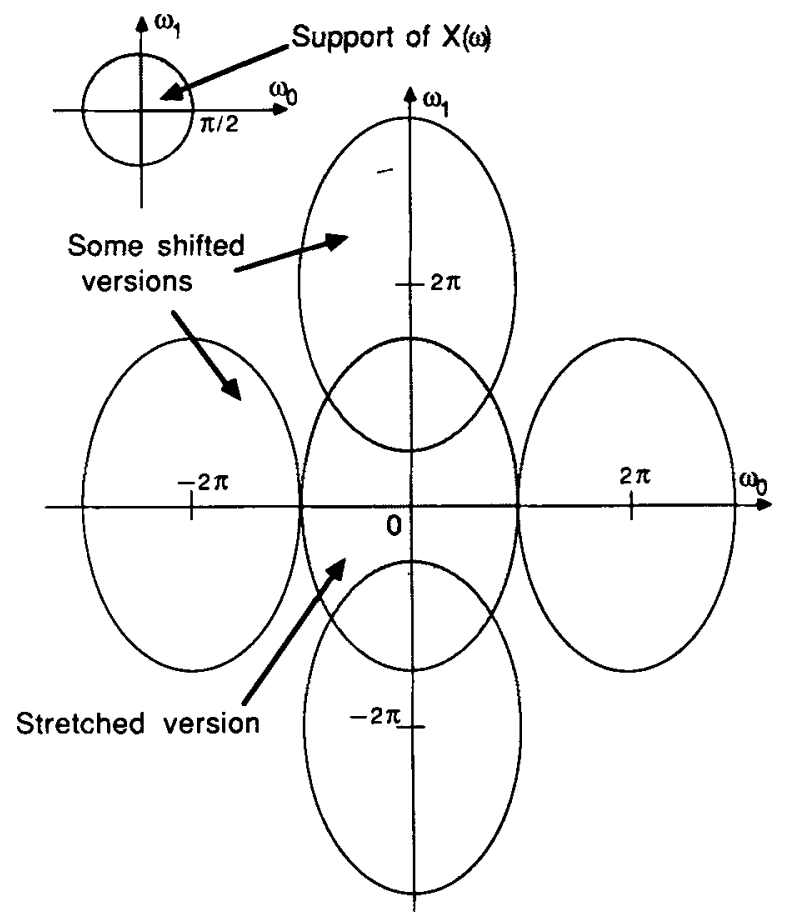

Figure 6. Demonstration of aliasing effects created by decimation.

version' $X\left(\mathbf{M}^{-T} \boldsymbol{\omega}\right)$. The remaining $J(\mathbf{M})-1$ terms with $\mathbf{k} \neq \mathbf{0}$ are the alias terms. These are shifted versions of the stretched version. Figure 6 demonstrates this idea for $\mathbf{M}=\left[\begin{array}{ll}2 & 0 \\ 0 & 3\end{array}\right]$. The support of $X(\omega)$, which is circular, gets stretched into an ellipse. The figure shows some of the neighbouring shifted versions also.

The decimator is said to create aliasing if any of the alias terms has overlap with the stretched version. We can recover $x(\mathbf{n})$ from the decimated version $y(\mathbf{n})$ if and only if there is no such overlap.

Range of summation in (19): The range over which the summation index $\mathbf{k}$ varies is not unique. In fact if we replace the phrase $k \in \mathcal{N}\left(\mathbf{M}^{T}\right)$ with $k \in \mathcal{N}\left(\mathbf{M}^{T} \mathbf{G}\right)$ for an arbitrary unimodular integer $\mathbf{G}$, the summation is unchanged. We omit the proof.

Interpolator. For the interpolator, the transform domain relation is easily verified to be

$$
Y(\omega)=X\left(\mathbf{M}^{T} \omega\right) \quad(\mathbf{M} \text {-fold interpolator })
$$

This means that there is no loss of information, and the spectrum merely gets transformed by the linear transformer $\mathbf{M}^{T}$. Since the periodicity matrix for $Y(\omega)$ is $2 \pi \mathbf{M}^{-T}$ rather than $2 \pi \mathrm{I}$, we have a total of $J(\mathbf{M})$ copies (or images) of the spectrum in $[0,2 \pi)^{D}$.

Figure 7 demonstrates this for hexagonal M. The support of $X(\omega)$ is assumed to be the diamond shown in figure 7a. Figure $7 \mathrm{~b}$ shows the support of the 'compressed 


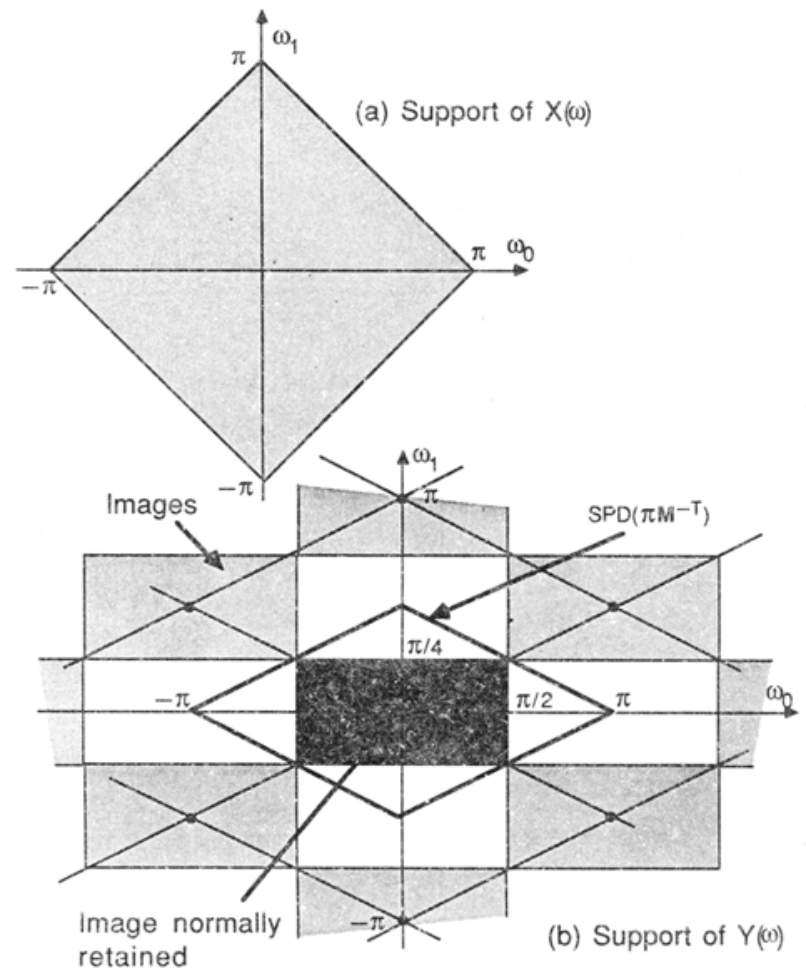

Figure 7. Demonstrating imaging due to $2-\mathrm{D}$ inierpolator.

version' $Y(\omega)$. The figure also shows a sketch of the lattice generated by the matrix

$$
2 \pi \mathbf{M}^{-T}=\frac{\pi}{2}\left[\begin{array}{rr}
2 & 1 \\
1 & -1
\end{array}\right] .
$$

(These are the intersections of the two sets of parallel lines shown). The fact that $2 \pi \mathbf{M}^{-T}$ is the periodicity matrix for $Y(\omega)$ is evident from the figure.

It is clear that if the interpolator is followed by a lowpass filter with passband support $\operatorname{SPD}\left(\pi \mathbf{M}^{-T}\right)$ (indicated in the figure), the extra images are eliminated, and only the main image (dark gray area) is retained. In the time domain this filtering is equivalent to replacing the zeros inserted by the interpolator with appropriate 'interpolated' values.

The notation $z^{i n}$ : Given the $D \times D$ integer matrix $\mathbf{M}$ and the $D \times 1$ vector $\mathbf{z}$, the notation $z^{(\mathbf{M})}$ stands for a $D \times 1$ vector whose $k$ th component is given by

$$
\left[\mathbf{z}^{(\mathbf{M})}\right]_{k} \triangleq z_{0}^{M_{a \cdot k}} z_{1}^{M_{1, k}} \ldots z_{D-1}^{M_{D}-1, k} .
$$

With this notation one can verify that the interpolator relation (22) is equivalent to

$$
Y_{z}(\mathbf{z})=X_{z}\left(\mathbf{z}^{(\mathbf{M})}\right) \quad(\mathbf{M} \text {-fold interpolator })
$$

To demonstrate the notation clearly, consider the hexagonal matrix $\mathbf{M}$ in (7). For this case we have

$$
\mathbf{z}^{(\mathbf{M})}=\left[\begin{array}{c}
z_{0} z_{1}^{2} \\
z_{0} z_{1}^{-2}
\end{array}\right] \quad \text { (hexagonal M). }
$$


(a)

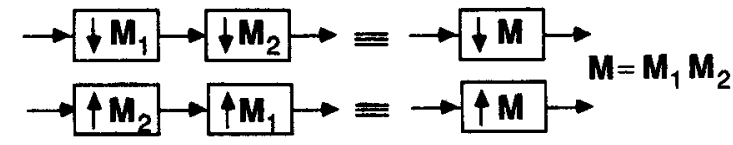

Figure 8. Cascade connections of multidimensional decimators and interpolators.

Unimodular decimators and interpolators: Suppose the decimation matrix $\mathbf{M}$ is unimodular, i.e., det $\mathbf{M}= \pm 1$. Since $J(\mathbf{M})=1$, all samples of $x(\mathbf{n})$ are retained, so that $y(\mathbf{n})$ is a permuted version of $x(\mathbf{n})$. The relation (19) now reduces to $Y(\boldsymbol{\omega})=X\left(\mathbf{M}^{-T} \boldsymbol{\omega}\right)$. By comparing with (22) we therefore conclude: $\mathbf{M}$-fold unimodular decimator is equivalent to $\mathbf{M}^{-1}$-fold unimodular interpolator! (The unimodularity of $\mathbf{M}$ assures that $\mathbf{M}^{-1}$ is also an integer matrix.)

\subsection{Cascade structures}

Figure 8a shows the cascade of two decimators $\mathbf{M}_{1}$ and $\mathbf{M}_{2}$, along with their equivalent. This equivalence can be verified using the decimator definition. Figure $8 b$ shows the cascade of two interpolators and their equivalent circuit, which is best verified using (22).

Noble identities: Figure 9 shows two useful multirate identities for multidimensional systems. Thus, if a transfer function $H_{z}(\mathrm{z})$ follows a decimator, we can move it to the left of the decimator provided we replace $\mathbf{z}$ with $z^{(\mathbf{M})}$ (i.e., replace $\omega$ with $\mathbf{M}^{T} \omega$ ). A similar identity for interpolators is also indicated in the figure. These are proved with the help of (19) and (22).

To demonstrate these consider the hexagonal decimator for which $\mathbf{z}^{(\mathbf{M})}$ is as in (25). The noble identity for the decimator is as in figure $10 \mathrm{a}$. Figures $10 \mathrm{~b}, \mathrm{c}$ show special cases of this. We can interpret figure $10 \mathrm{~b}$ as follows: let $x\left(n_{0}, n_{1}\right)$ be the input to the system. Then $\mathbf{M}$-fold decimation of $x\left(n_{0}+1, n_{1}+2\right)$ is the same as first decimating $x\left(n_{0}, n_{1}\right)$ by $\mathbf{M}$, and advancing only in the horizontal direction, i.e.,

$$
x\left(\mathbf{M n}+\left[\begin{array}{l}
1 \\
2
\end{array}\right]\right)=x\left(\mathbf{M}\left(n+\left[\begin{array}{l}
1 \\
0
\end{array}\right]\right)\right) .
$$

This is equivalent to the obvious statement that the 0th column of $\mathbf{M}$ is $\left[\begin{array}{l}1 \\ 2\end{array}\right]$ !

\subsection{Decimation and interpolation filters}

The decimation filter is a bandlimiting filter $H_{2}(\mathbf{z})$ that precedes the $\mathbf{M}$-fold decimator. Its purpose is to avoid aliasing. We prove that if the filter response is bandlimited to the region

$$
\boldsymbol{\omega}=\pi \mathbf{M}^{-T} \mathbf{x}+2 \pi \mathbf{m}, \quad \mathbf{x} \in[-1,1)^{D}, \quad \mathbf{m} \in \mathcal{N},
$$

then aliasing is avoided. The first term in the support is precisely the region $\operatorname{SPD}\left(\pi \mathbf{M}^{-T}\right)$, whereas the second term merely ensures the periodicity of $H(\omega)$. For

(a)

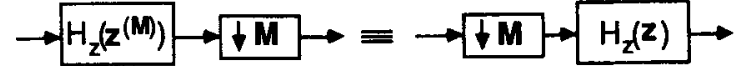

(b)

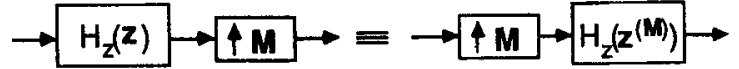

Figure 9. Noble identities for multidimensional decimators and interpolators. 
(a) $\rightarrow F_{z}\left(z_{0} z_{1}^{2}, z_{0} z_{1}^{-2}\right) \rightarrow+M \rightarrow \equiv \rightarrow+M,-F_{z}\left(z_{0}, z_{1}\right) \rightarrow$

(b)

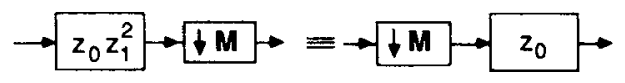

(c)

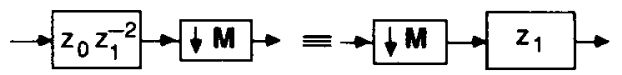

Figure 10. Demonstration of noble identity for hexagonal decimator.

the 2-D case it is instructive to express the filter support $\operatorname{SPD}\left(\pi \mathbf{M}^{-T}\right)$ explicitly in terms of the frequencies $\omega_{0}$ and $\omega_{1}$. This is given as

$$
-\pi \leqslant M_{00} \omega_{0}+M_{10} \omega_{1}<\pi, \quad-\pi \leqslant \mathbf{M}_{01} \omega_{0}+M_{11} \omega_{1}<\pi .
$$

Figure 11 shows this region for the hexagonal and quincunx decimators. Note that this region occupies one fourth of the area $[-\pi, \pi)^{2}$ for the hexagonal case and one half for quincunx case.

Proof that the support (27) avoids aliasing: Consider two terms in (19) with different values of $\mathbf{k}$, say $\mathbf{k}_{1}$ and $\mathbf{k}_{2}$. These terms have supports $\pi \mathbf{x}+2 \pi \mathbf{M}^{T} \mathbf{m}+2 \pi \mathbf{k}_{1}$ and $\pi \mathbf{x}+2 \pi \mathbf{M}^{T} \mathbf{m}+2 \pi \mathbf{k}_{2}$, where $\mathbf{x} \in[-1,1)^{D}$, and $\mathbf{m} \in \mathcal{N}$. Suppose there is overlap between the two regions, i.e., suppose there exist $\mathbf{x}_{1}, \mathbf{x}_{2} \in[-1,1)^{D}, \mathbf{m}_{1}, \mathbf{m}_{2} \in \mathcal{N}$, such that

Then

$$
\pi \mathbf{x}_{1}+2 \pi \mathbf{M}^{T} \mathbf{m}_{1}+2 \pi \mathbf{k}_{1}=\pi \mathbf{x}_{2}+2 \pi \mathbf{M}^{T} \mathbf{m}_{2}+2 \pi \mathbf{k}_{2} \text {. }
$$

$$
\mathbf{x}=\mathbf{M}^{T} \mathbf{m}+k_{1}-k_{2}, \quad m \in \mathscr{N},
$$

where $\mathbf{x}=0.5\left(\mathbf{x}_{2}-\mathbf{x}_{1}\right)$. Evidently $\mathbf{x} \in(-1,1)^{D}$. Since the right side is an integer, we must have $x=0$, i.e., $x_{1}=x_{2}$. Furthermore since $k_{i} \in \mathcal{N}\left(M^{T}\right)$ we can write $k_{i}=M^{T} y_{i}$ where $\mathbf{y}_{i} \in[0,1)^{D}$ so that (30) becomes $\mathbf{m}=\mathbf{y}_{2}-\mathbf{y}_{1} \in(-1,1)^{D}$. Since $\mathbf{m}$ is an integer this implies $\mathbf{m}=\mathbf{0}$, i.e., $\mathbf{y}_{1}=\mathbf{y}_{2}$, or $\mathbf{k}_{1}=\mathbf{k}_{2}$. Summarizing, if $\mathbf{k}_{1} \neq \mathbf{k}_{2}$ there cannot be an overlap.

$\nabla \nabla \nabla$

Note: One can verify that any shifted version of the support (27) is also sufficient, i.e., if the decimation filter is replaced with $H(\omega-\mathrm{c})$ for constant $\mathrm{c}$, it still avoids aliasing.

Interpolation filters: The purpose of interpolation filters is to suppress the $J(\mathbf{M})-1$ images created by the $\mathbf{M}$-fold interpolator. Once again, the lowpass support (27) will work, as demonstrated earlier in figure 7.

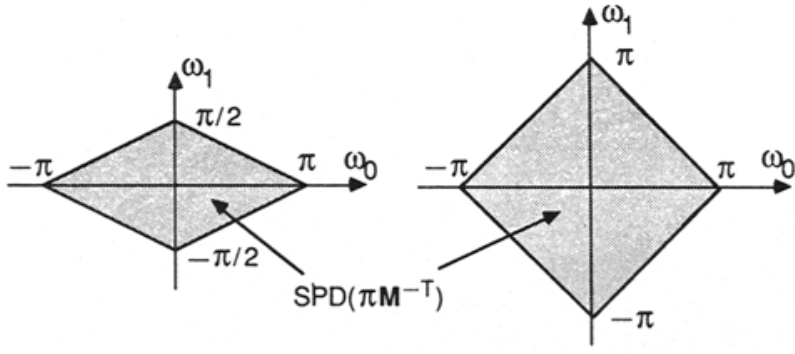

(a) Hexagonal

(b) Quincunx
Figure 11. Supports of ideal decimation filters for hexagonal and quincunx decimators. 


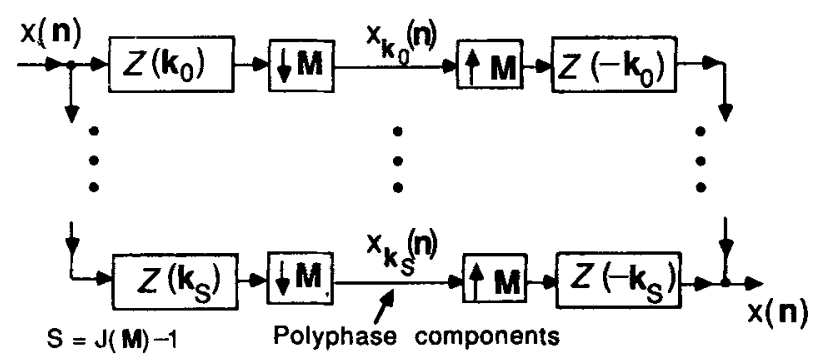

Figure 12. Generation of polyphase components, and recombination into the original signal.

\section{Polyphase decomposition}

We know that the time index $\mathbf{n}$ of a multidimensional sequence $x(\mathbf{n})$ can always be written as (15a). Based on this we define the kth polyphase component of $x(\mathbf{n})$ as

$$
x_{\mathbf{k}}(\mathbf{n})=x(\mathbf{M n}+\mathbf{k}), \quad \mathbf{k} \in \mathcal{N}(\mathbf{M}) .
$$

Evidently there are $J(\mathbf{M})$ polyphase components. $x_{\mathbf{k}}(\mathbf{n})$ is the $\mathbf{M}$-fold decimated version of the shifted version $x(\mathbf{n}+\mathbf{k})$. Figure 12 shows how $x_{\mathbf{k}}(\mathbf{n})$ is schematically generated. We can recover $x(\mathbf{n})$ from the set of signals $x_{\mathbf{k}}(\mathbf{n})$ by passing them through $\mathbf{M}$-fold interpolators, and interlacing the outputs as shown. Since $X_{z, k}(z)$ is the $\mathscr{Z}$-transform of $x_{\mathbf{k}}(\mathbf{n})$, we deduce the relation

$$
X_{z}(\mathbf{z})=\sum_{\mathbf{k} \in\{(\mathbf{M})} \mathscr{Z}(-\mathbf{k}) X_{z, \mathbf{k}}\left(\mathbf{z}^{(\mathbf{M})}\right) \quad \text { (Type } 1 \text { polyphase), }
$$

from this figure. In terms of the frequency variable $\omega$ this can be written as

$$
X(\omega)=\sum_{\mathbf{k} \in \mathbf{L},(M)} \exp \left[-j \omega^{T} \mathbf{k}\right] X_{\mathbf{k}}\left(\mathbf{M}^{T} \boldsymbol{\omega}\right) .
$$

Hexagonal $M:$ As an example consider again the hexagonal decimator. The $\operatorname{set} \mathcal{N}(\mathbf{M})$ is as in (14a). So the set of all 2-D integers are partitioned into four classes. Any integer $\mathbf{x}_{i}$ in the $i$ th class is such that $\mathbf{x}_{i} \bmod \mathbf{M}=\mathbf{k}_{i}$. Figure 13 demonstrates the partition. The elements $\mathscr{Z}(-\mathbf{k})$ are

$$
\mathscr{Z}\left(-\mathbf{k}_{0}\right)=1, \mathscr{Z}\left(-\mathbf{k}_{1}\right)=z_{0}^{-1}, \mathscr{Z}\left(-\mathbf{k}_{2}\right)=z_{0}^{-1} z_{1}^{-1}, \mathscr{Z}\left(-\mathbf{k}_{3}\right)=z_{0}^{-1} z_{1} .
$$

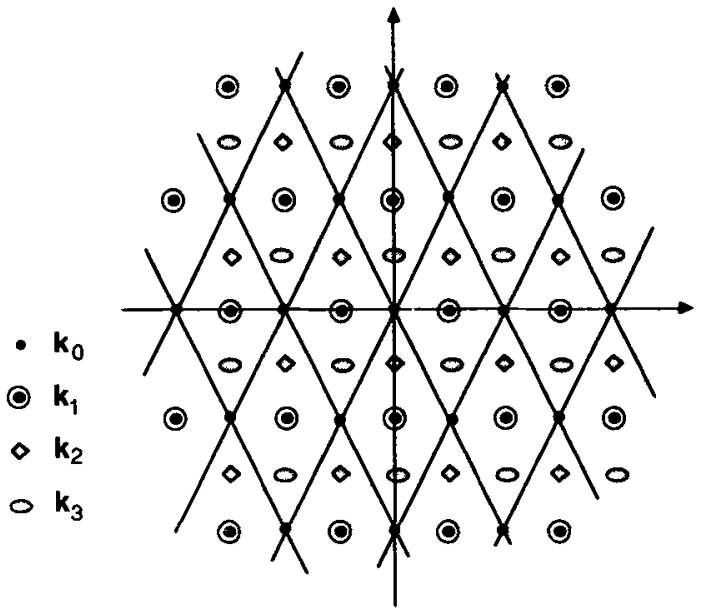

Figure 13. Demonstration of the four subsets of the partition generated in polyphase decomposition (hexagonal matrix). 


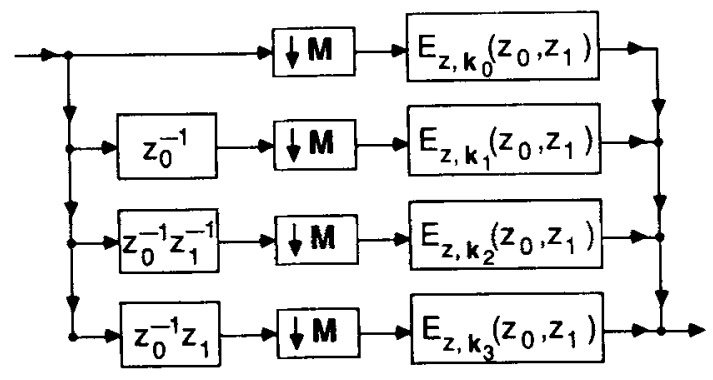

Figure 14. Polyphase implementation of decimation filter for hexagonal decimation.

With $\mathbf{z}^{(\mathbf{M})}$ given by (25) we thus obtain the polyphase decomposition

$$
\begin{aligned}
X_{z}\left(z_{0}, z_{1}\right)=X_{z, \mathbf{k}_{0}}\left(z_{0} z_{1}^{2}, z_{0} z_{1}^{-2}\right)+z_{0}^{-1} X_{z, \mathbf{k}_{1}}\left(z_{0} z_{1}^{2}, z_{0} z_{1}^{-2}\right)+ \\
+z_{0}^{-1} z_{1}^{-1} X_{z, \mathbf{k}_{2}}\left(z_{0} z_{1}^{2}, z_{0} z_{1}^{-2}\right)+z_{0}^{-1} z_{1} X_{z, \mathbf{k}_{3}}\left(z_{0} z_{1}^{2}, z_{0} z_{1}^{-2}\right)
\end{aligned}
$$

(hexagonal $\mathbf{M}$ ).

Figure 14 shows a decimation filter in polyphase form. Here the quantities $E_{z: \mathbf{k}}\left(z_{0} z_{1}^{2}\right.$, $z_{0} z_{1}^{-2}$ ) have been moved to the right of the decimators (by using the noble identity of figure 10a), so that they appear as $E_{z, \mathbf{k}_{i}}\left(z_{0} z_{1}\right)$. In this scheme the polyphase components are operating at the lower rate, resulting in improved computational efficiency.

Type 2 polyphase decomposition is obtained by defining the polyphase components to be $x_{\mathbf{k}}^{\prime}(\mathbf{n})=x(\mathbf{M n}-\mathbf{k})$, with $\mathbf{k} \in \mathcal{N}(\mathbf{M})$ so that

$$
X_{z}(\mathbf{z})=\sum_{\mathbf{k} \in \backslash(\backslash)} \mathscr{Z}(\mathbf{k}) X_{z, \mathbf{k}}^{\prime}\left(\mathbf{z}^{(\mathbf{M})}\right) \quad \text { (type } 2 \text { polyphase). }
$$

Type 1 decomposition is convenient for decimation filters and analysis-banks, whereas type 2 is more convenient for interpolation filters and synthesis-banks.

\section{Generalized DFT matrix}

The DFT matrix arises in many 1-D multirate systems, e.g., in the theory of QMF banks, in Nyquist filter theory, and in uniform DFT filter banks. An analogous matrix exists in the $D$-dimensional case, with very similar role and properties. We shall introduce this now.

\subsection{Generalized orthogonal exponentials}

Starting from our knowledge of polyphase components we shall first derive the so-called generalized exponentials, from which the generalized DFT matrix will follow. The polyphase component $x_{\mathbf{k}}(\mathbf{n})$ is the decimated version of $x(\mathbf{n}+\mathbf{k})$, so that $X_{\mathbf{k}}(\boldsymbol{\omega})$ can be expressed in terms of $X(\omega)$ using a relation like (19). Substituting into (33) we obtain

$$
X(\omega)=[1 / J(\mathbf{M})] \sum_{\mathbf{m} \in \mathcal{N}\left(\mathbf{M}^{T}\right)} X\left(\omega-2 \pi \mathbf{M}^{-T} \mathbf{m}\right) \sum_{\mathbf{k} \in \mathcal{N}(\mathbf{M})} \exp \left[-j 2 \pi \mathbf{m}^{T} \mathbf{M}^{-1} \mathbf{k}\right]
$$


From the fact that this relation holds for all possible functions $X(\omega)$, we can show

$$
\sum_{\mathbf{k} \in \mathcal{H}^{+}(\mathbf{M})} \exp \left[-j 2 \pi \mathbf{m}^{T} \mathbf{M}^{-1} \mathbf{k}\right]= \begin{cases}J(\mathbf{M}), & \mathbf{m}=\mathbf{0}, \\ 0, & \mathbf{m} \in \mathscr{N}\left(\mathbf{M}^{T}\right), \mathbf{m} \neq \mathbf{0} .\end{cases}
$$

Here $\mathbf{m}$ and $\mathbf{k}$ are $D$-component integers, and $\mathbf{M}$ is a $D \times D$ integer matrix. The reader can verify that the above equation can also be rearranged as

$$
\sum_{\mathbf{m} \in \mathcal{H}^{(}\left(\mathbf{M}^{T}\right)} \exp \left[-j 2 \pi \mathbf{m}^{T} \mathbf{M}^{-1} \mathbf{k}\right]= \begin{cases}J(\mathbf{M}), & \mathbf{k}=\mathbf{0}, \\ 0, & \mathbf{k} \in \mathscr{N}(\mathbf{M}), \mathbf{k} \neq \mathbf{0} .\end{cases}
$$

Generalized DFT matrix: We define the generalized DFT matrix $\mathbf{W}^{(g)}$ to be one whose elements are given by

$$
\left[\mathbf{W}^{(g)}\right]_{\mathbf{m}, \mathbf{k}}=\exp \left[-j 2 \pi \mathbf{m}^{T} \mathbf{M}^{-1} \mathbf{k}\right], \quad \mathbf{m} \in \mathcal{N}\left(\mathbf{M}^{T}\right), \mathbf{k} \in \mathcal{N}(\mathbf{M}) .
$$

Notice that the row and column indices for the matrix are taken as vectors $\mathbf{m}$ and k. Evidently this is a $J(M) \times J(M)$ matrix. In view of $(37), \mathbf{W}^{(s)}$ is unitary, satisfying

$$
\left[\mathbf{W}^{(g)}\right]^{\dagger} \mathbf{W}^{(g)}=J(\mathbf{M}) \mathbf{I}
$$

so that $\left[\mathbf{W}^{(g)}\right]^{-1}=\left[\mathbf{W}^{(g)}\right]^{\dagger} / J(\mathbf{M})$. Evidently this matrix reduces to the traditional DFT matrix when $M$ is scalar (1-D case).

To construct the matrix $\mathbf{W}^{(g)}$, the sets $\mathscr{N}(\mathbf{M})$ and $\mathscr{N}\left(\mathbf{M}^{T}\right)$ should first be identified. To illustrate, these sets are given in (14) for hexagonal $\mathbf{M}$. Computing the sixteen products $\mathbf{m}_{i}^{T} \mathbf{M}^{-1} \mathbf{k}_{l}$, we can verify

$$
\mathbf{W}^{(g)}=\left[\begin{array}{cccc}
1 & 1 & 1 & 1 \\
1 & W^{2} & W^{3} & W \\
1 & W^{4} & W^{6} & W^{2} \\
1 & W^{6} & W^{9} & W^{3}
\end{array}\right] \quad \text { (hexagonal M) }
$$

where $W \triangleq \exp [-j 2 \pi / 4]$. It turns out, therefore, that $\mathbf{W}^{(\boldsymbol{\theta})}$ is a column-permuted version of the traditional (1-D) $4 \times 4$ DFT matrix! This, however, is not a general fact. (For example, try the $2 \times 2$ matrix $M=2 I$.) It can in general be shown that $\mathbf{W}^{(g)}$ is a Kronecker product of an appropriate set of 'conventional' DFT matrices of smaller size.

\subsection{Uniform DFT filter banks}

One dimensional uniform DFT filter banks are described in Bellanger et al (1976) Crochiere \& Rabiner (1983) and Vaidyanathan (1990). In these systems, a set of $M$ filters is derived from a prototype $H_{0}(z)$ by use of the DFT matrix. All the filters are uniformly shifted versions of the prototype.

Figure 15a shows the multidimensional extension of this. This is a linear time invariant (LTI) system with one input and $J(\mathbf{M})$ outputs, and is characterized by the $J(\mathbf{M})$ transfer functions

$$
H_{\mathrm{m}}(\omega)=V_{\mathrm{m}}(\omega) / X(\omega), \quad \mathrm{m} \in \mathcal{N}\left(\mathbf{M}^{T}\right)
$$



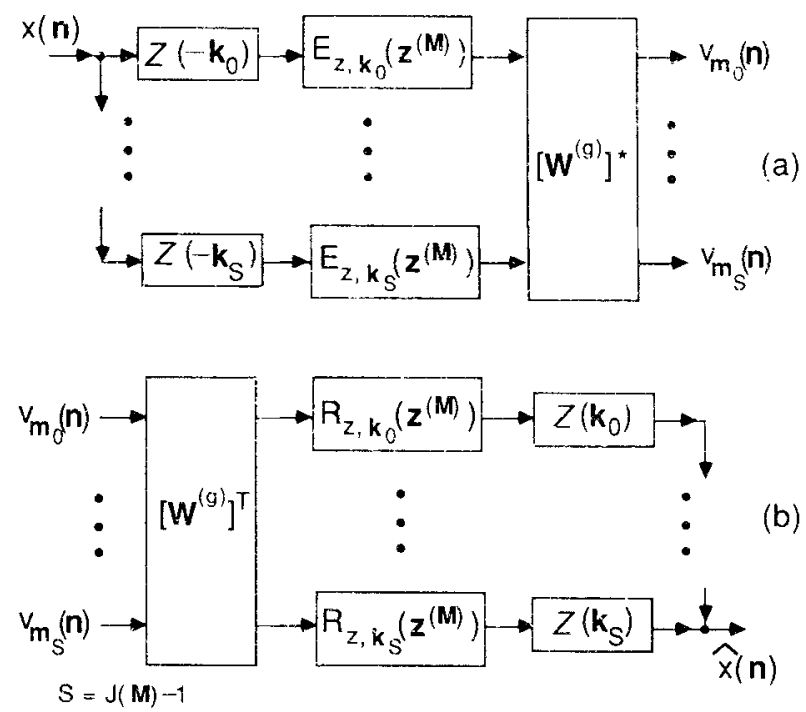

Figure 15. Uniform DF $\mathrm{T}$ filter banks. (a) Analysis bank, and (b) synthesis bank.

By using (39) for the entries of $\mathbf{W}^{(g)}$, we obtain

$$
\begin{aligned}
& H_{\mathbf{m}}(\boldsymbol{\omega})=\sum_{\mathbf{k} \in+1 \mathbf{M}} \exp \left[-j \boldsymbol{\omega}^{T} \mathbf{k}\right] \exp \left[j 2 \pi \mathbf{m}^{T} \mathbf{M}^{-1} \mathbf{k}\right] E_{\mathbf{k}}\left(\mathbf{M}^{T} \boldsymbol{\omega}\right) \\
& =\sum_{\mathbf{k} \in \mathcal{H}(\mathbf{M})} \exp \left[-j\left(\boldsymbol{\omega}-2 \pi \mathbf{M}^{-T} \mathbf{m}\right)^{T} \mathbf{k}\right] E_{\mathbf{k}}\left(\mathbf{M}^{T} \boldsymbol{\omega}\right) .
\end{aligned}
$$

$\left\{\right.$ Recall, according to our notations, $E_{2, k}\left(\mathbf{z}^{(\mathbf{M})}\right)=E_{\mathbf{k}}\left(\mathbf{M}^{T} \boldsymbol{\omega}\right)$ for steady state frequencies $\}$. Thus

$$
H_{\mathbf{m}}(\omega)=H_{\mathbf{m}}\left(\omega-2 \pi \mathbf{M}^{-T} \mathbf{m}\right), \quad \mathbf{m} \in \mathcal{1}\left(\mathbf{M}^{T}\right),
$$

where the prototype response is

$$
H_{\mathbf{m}_{\mathrm{u}}}(\boldsymbol{\omega})=\sum_{\mathbf{k} \in c_{1}(\mathbf{M})} \exp \left[-j \boldsymbol{\omega}^{T} \mathbf{k}\right] E_{\mathbf{k}}\left(\mathbf{M}^{T} \boldsymbol{\omega}\right) \quad \text { (Type } 1 \text { polyphase). }
$$

Equation (44) shows that the responses $H_{m}(\boldsymbol{\omega})$ are shifted versions of the response $H_{\mathbf{m}_{1}}(\omega)$. The shifted locations are the points on the scaled reciprocal lattice corresponding to $\mathbf{M}$ So the system of figure 15 a represents a multidimensional uniform-DFT analysis bank. Evidently $J(\mathbf{M})$ filters are obtained at the cost of one filter plus the overhead to implement the matrix $\left[\mathbf{W}^{(g)}\right]^{*}$.

Next, figure $15 \mathrm{~b}$ represents a uniform DFT synthesis bank. The synthesis filters $F_{\mathrm{m}_{l}}(\omega)$ are transfer functions from $v_{\mathbf{m}}(\mathbf{n})$ to $\hat{x}(\mathbf{n})$. These are related exactly as in (44) (with $H$ replaced by $F$ everywhere), with prototype $F_{p=1}(\omega)=\Sigma_{\mathbf{k} \in(\mathbf{M})} \exp \left[j \omega^{T} \mathbf{k}\right] R_{\mathbf{k}}\left(\mathbf{M}^{T} \omega\right)$ (which is in type 2 polyphase form).

Consider the example of hexagonal $\mathbf{M}$. The eiements of the set.1 $\left(\mathbf{M}^{T}\right)$ are given in (14b), so that the shift vectors $2 \pi \mathbf{M}^{-T} \mathbf{m}$ in (44) are

$$
\left[\begin{array}{l}
0 \\
0
\end{array}\right], \pi\left[\begin{array}{c}
1 \\
0.5
\end{array}\right], \pi\left[\begin{array}{l}
0 \\
1
\end{array}\right], \quad \pi\left[\begin{array}{c}
1 \\
1.5
\end{array}\right] .
$$

Assume that the prototype filter $H_{m}(\boldsymbol{\omega})$ is lowpass with passband support as indicated 


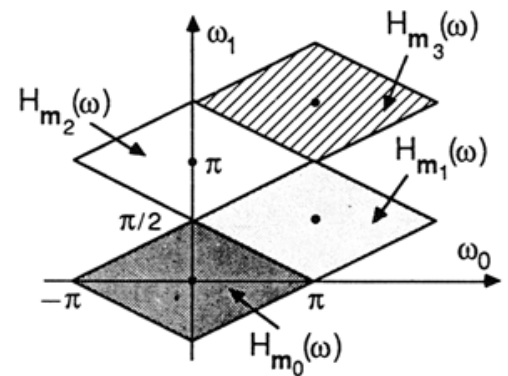

Figure 16. Example of supports of the four filters in a uniform DFT bank.

in figure 16 [which is $\left.\operatorname{SPD}\left(\pi \mathbf{M}^{-T}\right)\right]$. Then the three shifted filters have supports as shown in the same figure. If we keep in mind the fact that each of the responses in this figure is periodic (periodicity matrix $2 \pi \mathrm{I}$ ), we can verify that the four passbands (which are disjoint) fill the entire frequency plane. If the prototype filter had some other kind of support (inconsistent with our choice of $\mathbf{M}$ ), this would not be true.

\section{Maximally decimated filter banks}

Figure 17 shows a filter bank system with $\mathbf{M}$-fold decimators and interpolators. Recall that each decimator reduces sample density by $J(\mathbf{M})=|\operatorname{det} \mathbf{M}|$. Since there are $J(\mathbf{M})$ analysis filters, the overall analysis bank preserves the same original sampling density. We say that the system is maximally decimated because a higher decimation ratio (with the same number of filters) would lead to loss of information. Note that the subscripts on the filters are integer vectors $\mathbf{k}$, with $k \in \mathcal{N}\left(\mathbf{M}^{T}\right)$. This is merely a matter of convenience.

This system is commonly used in subband coding of images (Vetterli 1984; Wachersreuther 1986; Woods \& O'Neil 1986; Ansari \& Lau 1987; Vaidyanathan 1987; Viscito \& Allebach 1988; Ansari \& Guillemot 1990; Bamberger \& Smith 1990; Karlsson \& Vetterli 1990). Each decimated subband signal (i.e., analysis filter output) is quantized (or encoded using sophisticated techniques, e.g., vector quantization) based on the amount of subband energy and other subjective considerations. The result is an overall reduction in the number of bits per pixel required to represent the original image. We shall refrain from providing more precise details on coding, which can be found in Jayant \& Noll (1984). In what follows, we are interested in the distortion introduced by the filters and decimators.

Typical decimation matrices used for subband coding are the hexagonal and quincunx matrices given in (7). Assume for example that $\mathbf{M}$ is hexagonal so that $J(M)=4$. One (of the many possible) choice for the four analysis filter responses is given in figure 16. Assume for the moment that the analysis filters are ideal (i.e.,

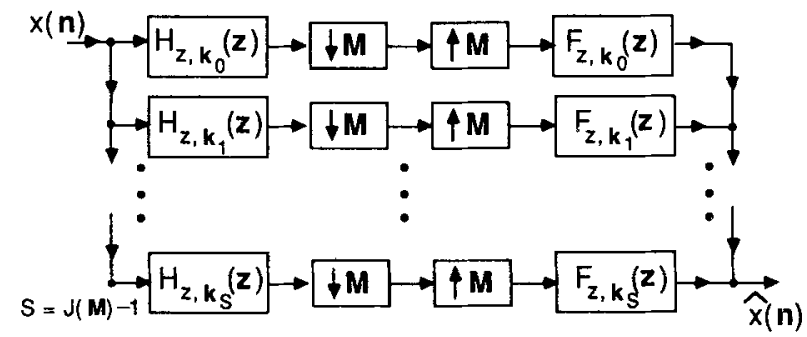

Figure 17. The multidimensional maximally decimated filter bank. 
response equals unity in passband and zero in stopband), and the synthesis filters are chosen as $F_{z=\mathbf{k}}(\mathbf{z})=H_{z, \mathbf{k}}(\mathrm{z})$. By using the results of $\S 4$ we can then verify that $\hat{x}(\mathbf{n})=x(\mathbf{n})$, i.e., we have a perfect reconstruction system.

\subsection{Alias-component matrix formulation}

In practice the filters are not ideal so that decimation creates aliasing. The signal $\hat{X}(\omega)$ is therefore a linear combination of $X(\omega)$ with shifted copies $X\left(\omega-2 \pi \mathbf{M}^{-T} \mathrm{~m}\right)$ (which are the alias-components). More quantitatively, it can be shown that

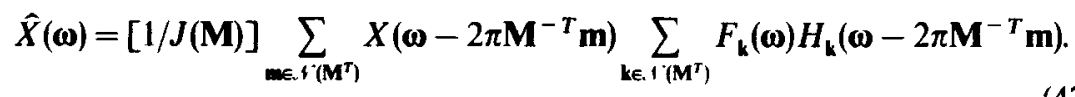

[Notice, according to our notations, $F_{z, \mathbf{k}}(\mathbf{z})$ becomes $F_{\mathbf{k}}(\omega)$ for steady state frequencies.] The alias-components can be eliminated if the filters are chosen such that

$$
\sum_{\mathbf{k} \in \mathcal{H}\left(\mathbf{M}^{r}\right)} F_{\mathbf{k}}(\boldsymbol{\omega}) H_{\mathbf{k}}\left(\boldsymbol{\omega}-2 \pi \mathbf{M}^{-T} \mathbf{m}\right)=0, \quad \mathbf{m} \neq \mathbf{0}, \quad \mathbf{m} \in \mathcal{N}\left(\mathbf{M}^{T}\right)
$$

Under this alias-free condition we have

$$
\hat{X}(\omega)=\underbrace{\frac{1}{J(\mathbf{M})} \sum_{\mathbf{k} \epsilon_{*}+\left(\mathbf{M}^{T}\right)} F_{\mathbf{k}}(\omega) H_{\mathbf{k}}(\omega) X(\omega)}_{T(\omega)}
$$

with $T(\omega)$ representing the overall distortion function. If $T(\omega)$ is allpass the system is free from amplitude distortion; if $T(\omega)=c \exp \left[-j \omega^{T} n_{0}\right]$ for some integer $n_{0}$, the system has perfect reconstruction property, i.e., $\hat{x}(n)=c x\left(n-n_{0}\right)$. The condition for alias cancellation can be written in the form

$$
\frac{\mathbf{H}(\boldsymbol{\omega}) \mathbf{f}(\boldsymbol{\omega})}{J(\mathbf{M})}=\left[\begin{array}{c}
X \\
0 \\
\vdots \\
0
\end{array}\right]
$$

where $\mathbf{H}(\boldsymbol{\omega})$ is the alias-component (AC) matrix with elements $[H(\omega)]_{\mathrm{m}, \mathrm{k}}=$ $H_{\mathrm{k}}\left(\omega-2 \pi \mathbf{M}^{-T} \mathrm{~m}\right)$, and $\mathrm{f}(\omega)$ is the synthesis filter vector with elements $[\mathrm{f}(\omega)]_{\mathrm{k}}=F_{\mathrm{k}}(\omega)$. Given the set of analysis filters [and hence the matrix $\mathbf{H}(\omega)$ ], we can therefore find synthesis filters for alias-cancellation by solving (50) (subject to invertibility of $\mathbf{H}(\omega)$ ).

To illustrate the AC matrix, consider again the hexagonal decimator (7). Using the elements in (14b) the four vectors $x_{i}=M^{-T} m_{i}$ can be calculated, and the AC matrix is verified to be

$$
\left[\begin{array}{cccc}
H_{z, \mathbf{k}_{0}}\left(z_{0}, z_{1}\right) & H_{z, \mathbf{k}_{1}}\left(z_{0}, z_{1}\right) & H_{z, \mathbf{k}_{2}}\left(z_{0}, z_{1}\right) & H_{z, \mathbf{k}_{3}}\left(z_{0}, z_{1}\right) \\
H_{z, \mathbf{k}_{0}}\left(-z_{0},-j z_{1}\right) & H_{z, \mathbf{k}_{1}}\left(-z_{0},-j z_{1}\right) & H_{z, \mathbf{k}_{2}}\left(-z_{0},-j z_{1}\right) & H_{z, \mathbf{k}}\left(-z_{0},-j z_{1}\right) \\
H_{z, \mathbf{k}_{0}}\left(z_{0},-z_{1}\right) & H_{z, \mathbf{k}_{1}}\left(z_{0},-z_{1}\right) & H_{z, \mathbf{k}_{2}}\left(z_{0},-z_{1}\right) & H_{z, \mathbf{k}_{3}}\left(z_{0},-z_{1}\right) \\
H_{z, \mathbf{k}_{0}}\left(-z_{0}, j z_{1}\right) & H_{z, \mathbf{k}_{1}}\left(-z_{0}, j z_{1}\right) & H_{z, \mathbf{k}_{2}}\left(-z_{0}, j z_{1}\right) & H_{z, \mathbf{k}_{3}}\left(-z_{0}, j z_{1}\right)
\end{array}\right]
$$




\subsection{Polyphase decomposition and perfect reconstruction}

As in the 1-D case it is possible to avoid inversion of the AC matrix, if the filter bank is represented in polyphase form. This simplifies the alias cancellation and perfect reconstruction problems both conceptually and from a design viewpoint. This idea has received more attention recently (Vetterli 1984; Wachersreuther 1986; Ansari \& Lau 1987; Vaidyanathan 1987; Viscito \& Allebach 1988; Ansari \& Guillemot 1990; Bamberger \& Smith 1990; Karlsson \& Vetterli 1990) than the topics we discussed earlier. So we shall only give a brief exposition.

Express the analysis and synthesis filters in type 1 and type 2 polyphase forms respectively:

$$
H_{z, \mathbf{k}}(\mathbf{z})=\sum_{\mathbf{m} \in I^{\prime}(\mathbf{M})} \mathscr{Z}(-\mathbf{m}) E_{z, \mathbf{k}, \mathbf{m}}\left(\mathbf{z}^{(\mathbf{M})}\right), \quad F_{z, \mathbf{k}}(\mathbf{z})=\sum_{\mathbf{m} \in \jmath^{\prime}(\mathbf{M})} \mathscr{Z}(\mathbf{m}) R_{z, \mathbf{m}, \mathbf{k}}\left(\mathbf{z}^{(\mathbf{M})}\right)
$$

We can then redraw the system as in figure $18 \mathrm{a}$ where $\mathbf{E}_{\mathbf{z}}(\mathbf{z})$ and $\mathbf{R}_{z}(\mathbf{z})$ are $J(\mathbf{M}) \times J(\mathbf{M})$ polyphase matrices with elements given by

$$
\left[\mathbf{E}_{z}(\mathbf{z})\right]_{\mathbf{k}, \mathbf{m}} \triangleq E_{z, \mathbf{k}, \mathbf{m}}(\mathbf{z}), \quad\left[\mathbf{R}_{\mathbf{z}}(\mathbf{z})\right]_{\mathrm{m}, \mathbf{k}} \triangleq \mathbf{R}_{z, \mathbf{m}, \mathbf{k}}(\mathbf{z})
$$

By use of noble identities (figure 9) this can further be redrawn as in figure $18 \mathrm{~b}$.

Perfect reconstruction using lossless matrices: We know that figure 12 represents a perfect reconstruction system. It can be verified that if $\mathbf{k}_{i}$ is replaced by $-\mathbf{k}_{i}$ everywhere, this property remains true. So we conclude that if $\mathbf{R}_{z}(\mathbf{z})=\mathbf{E}_{z}^{-1}(\mathbf{z})$, the structure of figure $18 \mathrm{~b}$ has perfect reconstruction property. For example assume that the analysis filters are FIR so that the matrix $\mathbf{E}_{\mathbf{z}}(\mathbf{z})$ has polynomial entries. Assume further that this matrix is forced to be lossless (Vaidyanathan 1990). This means that $\mathbf{E}(\omega)$ is
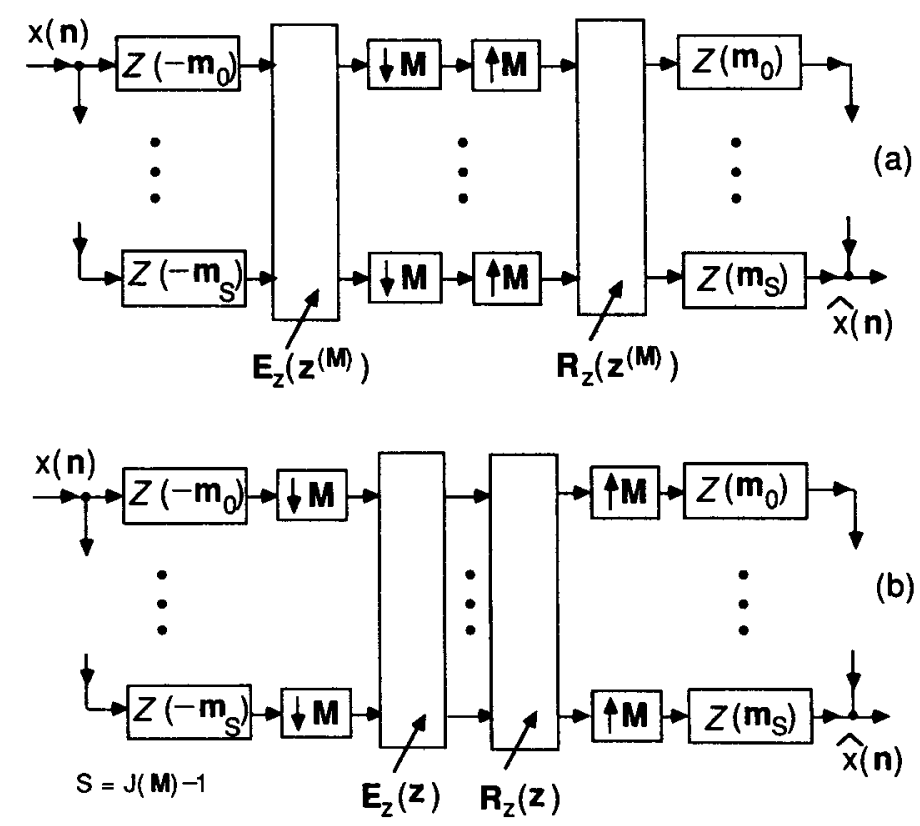

Figure 18. Polyphase representations of multidimensional maximally decimated filter bank. 


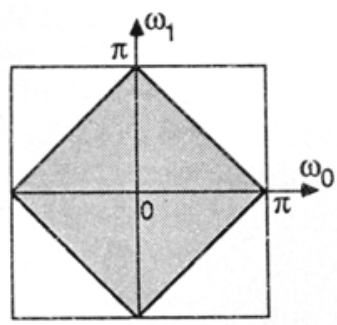

(a) Lowpass filter

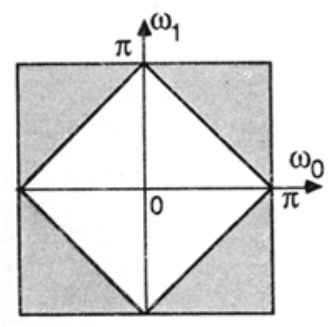

(b) Highpass filter
Figure 19. Examples of analysis-filter supports for 2-D quincunx QMF bank. Shaded regions indicate passbands.

unitary, i.e., $\mathbf{E}^{\dagger}(\omega) \mathbf{E}(\omega)=c$ I for all $\boldsymbol{\omega}$ (with $c=$ positive constant). By choosing $\mathbf{R}(\boldsymbol{\omega})=\mathbf{E}^{\dagger}(\omega)$ we therefore obtain perfect reconstruction. Assuming that all filters have real coefficients, this choice of $\mathbf{R}(\omega)$ is equivalent to

$$
\mathbf{R}_{z}\left(z_{0}, \ldots z_{D-1}\right)=\mathbf{E}_{-}^{T}\left(z_{0}^{-1}, \ldots z_{D-1}^{-1}\right) .
$$

Under this condition the synthesis filters can be found from analysis filters by inspection, as they are related as $F_{z, \mathbf{k}}\left(z_{0}, \ldots, z_{D-1}\right)=H_{z, \mathbf{k}}\left(z_{0}^{-1}, \ldots, z_{D-1}^{-1}\right)$.

Forcing lossless property: Even though the most general structure for multidimensional FIR lossless matrices has not been found, cascades of simple lossless building blocks have been used to obtain $\mathbf{E}_{z}(\mathbf{z})$. By optimizing the multipliers in these structures it is possible to obtained desired shapes for the frequency responses of analysis filters.

Comment on quincunx case: Notice that for the quincunx case there are two analysis filters. It is typical to take $H_{z, \mathbf{k}_{0}}(\mathbf{z})$ to be diamond shaped lowpass and $H_{z, \mathbf{k}_{1}}(\mathbf{z})$ to be its complement (as indicated in figure 19). The design of such filters starting from a 1-D prototype is described in Ansari \& Lau (1987). In particular, a technique is described in Ansari \& Guillemot (1990), whereby $H_{z, \mathbf{k}_{0}}(\mathbf{z})$ is designed to be a spectral factor of a diamond shaped zero phase filter. This permits design of an FIR perfect reconstruction quincunx QMF bank. More direct design of such systems based on lossless $\mathbf{E}_{z}(\mathbf{z})$ can be found (Karlsson \& Vetterli 1990).

\section{Concluding remarks}

Even though the theory of multidimensional multirate systems based on generalized decimation matrices is algebraically very elegant and powerful, it might give the first impression of being more complicated than for the 1-D case. However, efficient use of notations and definitions greatly simplifies the understanding of these systems. We therefore conclude with a summary of these.

Work supported in parts by National Science Foundation grants MIP 8919196, MIP 8604456, DCI 8552579, and matching funds from Hughes Aircraft Co., and Tektronix, Inc. 


\section{List of special notation}

$\boldsymbol{\omega}=\left[\omega_{0} \ldots \omega_{D-1}\right]^{T}, \mathbf{z}=\left[z_{0} \ldots z_{D-1}\right]^{T}, \mathbf{n}=\left[n_{0} \ldots n_{D-1}\right]^{T}$

$\mathcal{N}$ : set of all $D \times 1$ integer vectors.

M: real nonsingular $D \times D$ matrix.

$[a, b)^{D}$ : set of all real $D \times 1$ vectors $\mathbf{x}$ with components $x_{i}$ in $a \leqslant x_{i}<b$.

$L A T(\mathbf{M})$ : lattice generated by $\mathbf{M}$ (called integer lattice if $\mathbf{M}$ is integer).

FPD(M) (fundamental parallelopiped): set of $D \times 1$ vectors $\mathbf{M x}$ with $\mathbf{x} \in[0,1)^{D}$.

$\operatorname{SPD}(\mathbf{M})$ (symmetric parallelopiped): set of $D \times 1$ vectors $\mathbf{M x}$ with $\mathbf{x} \in[-1,1)^{D}$.

$\mathscr{N}(\mathbf{M})$ : set of integer vectors in FPD(M) (M being an integer matrix).

$\mathbf{z}^{(\mathbf{M})}: D \times 1$ vector with $\left[\mathbf{z}^{(\mathbf{M})}\right]_{k}=z_{0}^{\mathbf{M}_{\mathbf{0}, k}} \cdots z_{D}^{M_{0}-1, k}$. This occurs in transform domain description of interpoiator, and is also useful in polyphase decompositions (32), (35). $J(\mathbf{M})=|\operatorname{det} \mathbf{M}|=$ volume of $\operatorname{FPD}(\mathbf{M})$. For integer $\mathbf{M}, J(\mathbf{M})=$ number of elements in $\mathscr{N}(\mathbf{M})$.

\section{References}

Ansari R, Guillemot C 1990 Exact reconstruction filters banks using diamond filters. Proc. Bilkent Int. Conf. Commun. Control and Signal Process. pp. 1412-1424

Ansari R, Lau C-L 1987 Two-dimensional IIR filters for exact reconstruction in tree structured sub-band decomposition. Electron. Lett. 23: 633-634

Bamberger R H, Smith M J T 1990 Efficient 2-D analysis/synthesis filler banks for directional image component representation. Proc. IEEE Int. Conf. Circuits and Syst. pp. 2009-2012

Bellanger M, Bonnerot G, Coudreuse M 1976 Digital filtering by polyphase network: application to sample rate alteration and filter banks. IEEE Trans. Acoust. Speech Signal Process. 24: 109-114

Cassels J W S 1959 An introduction to the geometry of numbers (Berlin: Springer Verlag)

Crochiere R E 1977 On the design of sub-band coders for low bit rate speech communication. Bell Syst. Tech. 56: 747-771

Crochiere R E, Rabiner L R 1981 Interpolation and decimation of digital signals: a tutorial review. Proc. IEEE 69: 300-331

Crochiere R E, Rabiner L R 1983 Multirate digital signal processing (Englewood Cliff, NJ: Prentice Hall)

Dubois $\mathrm{E} 1985$ The sampling and reconstruction of time-varying imagery with application in video systems. Proc. IEEE 73: 502-522

Dudgeon D E, Mersereau R M 1984 Multidimensional digital signal processing, (Englewood Cliffs, NJ: Prentice Hall)

Fettweis A, Leickel T, Bolle M, Sauvagerd U 1990 Realization of filter banks by means of wave digital filters. Proc. IEEE Int. Conf. Circuits Sysm. pp. 2013-2016

Jayant N S, Noll P 1984 Digital coding of waveforms (Englewood Cliffs, NJ: Prentice Hall)

Karlsson G, Vetterli M 1990 Theory of two-dimensional multirate filter banks. IEEE Trans. Acoust. Speech Signal Process. ASSP: 925-937

Oppenheim A V, Schafer R W 1975 Digital signal processing (Englewood Cliffs, NJ: Prentice Hall)

Vaidyanathan P P 1987 Perfect reconstruction QMF banks for two-dimensional applications. IEEE Trans. Circuits Syst. 34: 976-978

Vaidyanathan P P 1990 Multirate digital filters, filter banks, polyphase networks, and applications. Proc. IEEE 78: 56-93

Vaidyanathan P P, Doganata Z 1989 The role of lossless systems in modern digital signal processing. A tutorial. Special issue on Circuits and systems, IEEE Trans. on Education 32: 181-197

Vetterli M 1984 Multi-dimensional sub-band coding: some theory and algorithms. Signal Process. 6: 97-112

Viscito E, Allebach J 1988 Design of perfect reconstruction multi dimensional filter banks using cascaded Smith-form matrices. Proc. of the IEEE Int. Symp. on Circuits Systems, Espoo, Finland, pp. 831-834

Wackersreuther G 1986 On two-dimensional polyphase filter banks. IEEE Trans. Acoust., Speech Signal Process. 34: 192-199

Woods J W, O'Neil S D 1986 Subband coding of images. IEEE Trans. Acoust. Speech and Signal Process. 34: $1278-1288$ 